\title{
Pádel: una mirada compleja, dinámica y no lineal en la iniciación deportiva y el entrenamiento \\ Paddle-tennis: a complex, dynamic and non-linear approach for teaching- learning processes and training \\ Enrique Lacasa Claver, Cristòfol Salas Santandreu, CarlotaTorrents Martin \\ Universidad de Lérida (España)
}

\begin{abstract}
Resumen: En contraposición a perspectivas tradicionales, reduccionistasy excesivamente mecanicistas, consideramosalas/ los jugadoras/ es de pádel como sistemas dinámicos y complejos, y a sus procesos de adquisición de habilidades como no lineal es. El objetivo de este artículo es el de presentar las consecuencias de la introducción de las ciencias de la complejidad en el entrenamiento y el aprendizaje y mostrar aplicaciones concretas en el pádel. Tras una aproximación a conceptos clave como Constraints-led Approach, constreñimiento, auto-organización o ciclo percepción-acción, mostramos dos estudios piloto aplicables al entrenamiento del pádel donde se modifica el comportamiento a partir de la manipulación de constreñimientos delatarea: a) Un juego reducido queescal ael espacio y equipamiento para adaptarlo ajugadoras y jugadores benjamines de una escuela de pádel. Al comparar el comportamiento de las niñas y niños en juego adaptado vs. juego reglamentario, se observó que se producíaun aumento de oportunidades en cuanto al número y variedad deacciones; b) El Cruzadito, un juego reducido que limita el espacio y el número dejugadores. Secomparó, en tres grupos de edad, la carga detrabajo de la situación reducida vs. juego reglamentario. Se comprobó que la intensidad fue mayor durante la práctica en el juego reducido. A partir de estos resultados, se discute sobre los beneficios de mejorar el comportamiento de los jugadores y jugadoras estimulando aspectos condicionales sin necesidad de aislar al jugador de los acoplamientos percepción-acción claves y específicos del pádel. Sugerimos que la perspectiva compleja, dinámica y no-lineal puede ser un marco idóneo para el diseño de escenarios de aprendizaje y mejora integral de deportistas.
\end{abstract}

Palabras clave: complejidad, dinámica ecológica, auto-organización, emergencia, aprendizaje guiado por constreñimientos.

Summary: In contrast to traditional, reductionist and excessively mechanistic approaches, we propose that padel players are complex dynamical systems and their skill acquisition processes are non-linear. The aim of this paper is to describe the consequences of the introduction of complex systems sciences to sport training and to show two practical proposals. Some key concepts such as Constraints-led Approach, constraint, self-organization or perception-action are explained before presenting two pilot studies to modify the behavior by means of the manipulation of task constraints: a) A small sided game that scales the space and equipment to adapt it to young players of ajunior school. By comparing the behavior of the girls and boys in the adapted game vs, regular game, it issuggested that thereare more oppor tunities for greater number and variety of actions when reducing the game. B) Cruzadito is a small-sided game that limits the space and the number of players and it has been applied to three age groups. By comparing the workload of the small-sided situation vs. regular game, it is suggested that physical load can even be increased. The benefits of these proposals are discussed, considering the differences with approaches based on isolating the player from the key and specific perception-action links of padel. It is suggested that the complex, dynamic and non-linear perspective can be an excellent approach for designing learning contexts and improving athlete's states.

Key words: Padel, complexity, ecological dynamics, self-organization, emergence, constraint-led approach.

\section{Introducción}

«iU mm! ¡Q ué bien me quedó esta bola! Pero esta mos a la altura del mar. Aunque con este calor... No. Esta pista está muy lenta.» Daniel «Sanyo» Gutiérrez. JugadorW orld Padel Tour en Spot publicitario Pelotas Head SPro, 2019.

Fecha recepción: 17-06-20. Fecha de aceptación: 05-01-21 Enrique Lacasa Claver

qlacasa@inefc.es
No hace falta escuchar a un jugador profesional acerca de la multitud de variables, de índole muy diversa, que van a condicionar las acciones de juego. Cualquier curioso que se inicie en este atractivo deporte lo va a constatar con rapidez. No obstante, perspectivas reduccionistas siguen siendo mayoritarias en el entrenamiento de un deporte como el pádel que requiere la constante interacción con un entorno cambiante e imprevisible.

Paracomprender los entresijos de una acción de jue- 
go (y por ende de los aprendizajes o del entrenamiento de esa determinada habilidad deportiva) se necesitará una visión amplia acerca de cómo se coordinan los diferentes agentes que participan. Esta se al eja de la visión dualista, imperante aún hoy en día, de separación entre cuerpo y mente y de que la percepción es previa y antecede (en una secuencia lineal) a la acción 0 al movimiento (Aivar \& Ramón, 2006). Esa dualidad asume que el cerebro, a modo de director de orquesta, escoge cuál es la mejor opción dentro de un repertorio aprendido.

Debido a esta visión, tradicionalmente se ha acepta do que el aprendizaje de la técnica debe ser un paso previo a la introducción de los aspectos tácticos o de los principiosoperativos del juego (M itchell, 0 slin \& Griffin, 2006). Habitualmente, los manuales de técnica proponen modelos de enseñanza que priorizan la adquisición de los elementos técnicos de forma lineal, ordenada, aislada y descontextualizada de las exigencias reales de la práctica. Esto tiene como consecuencia que abunden las situaciones de entrenamiento basadas en la repetición de ejercicios y en la corrección de la ejecución de un gesto técnico (considerando que hay un gesto ideal independientemente de las fuentes de información disponibles). Estas correcciones o instrucciones guiarán las intenciones, percepcionesy acciones de juego (H eadrick, Renshaw, Davids, Pinder \& Araújo, 2015). Ese tipo de propuestas obvian las investigaciones que muestran que percibimos la realidad a través de una interacción diná mica con el entorno, con lo que percepción y acción constituyen un único proceso entre la acción de la persona que juega y la información del entorno (Gibson, 1979; Heras, 2012). Así mismo, obvian que las personas, como todo sistema vivo, somos sistemas complejos adaptativos, con infinidad de variables que interactúan y con la capacidad de autoorganizarse y aprender.

Los factores que aparecían en la cita con que se inicia la introducción (la altitud, el calor, la lentitud de la moqueta) constituyen en ese momento concreto del juego la información del entorno que constriñe el comportamiento del jugador en cuestión. A partir de la propuestade N ewell (1986) y delaactualización de Balagué, Pol, Ric, Torrents y Hristovski (2019), consideramos que los constreñimientos que influyen en el comporta miento se pueden dividir en: del organismo (personal, individual) 0 del medio (entorno, ambiente). A partir de la interacción de estos dos tipos de constreñimientos emergerán los constreñimientos de la tarea, que dependerán de las especificaciones que se hagan al entorno (incluidas las instrucciones o las acciones de otros participantes) y las intenciones y disponibilidad de la persona que va a realizar la tarea (se requiere que la persona quieray/ o pueda realizar la tarea para que realmente las propuestas del entorno constriñan el comportamiento de esa persona). Los constreñimientos de la cita serán propios del entorno, mientras que el nivel del jugador, que es lo suficientemente elevado como para que ese tipo de factores constriñan su juego, será un constreñimiento del organismo o personal, así como su antropometría, la motivación, el control de las emociones o sus intenciones. En cuanto a los de la tarea, en este caso serán las reglas específicas del pádel que se apliquen, el tipo y balance de la pala, o la presión de la pelota, entre otros (Passos, Araújo, Davids \& Shuttleworth, 2008). Todos estos aspectos, interrelacionados en todos los niveles y escalas temporales (Balagué et al., 2019), van a constreñir el juego de maneraqueel jugador organiza su comportamiento para resolver los problemas que, de forma dinámica, se le presentan (Davids, Button \& Bennett, 2008). Este proceso se relaciona con un principio fundamental de los sistemas complejos como es la emergencia espontánea del comportamiento en base a la capacidad de autoorganización que posee todo sistema complejo adaptativo (Chow, Davids, Hristovski, Araújo \& Passos, 2011).

El pádel no es un deporte individual. Se coopera con otra persona y se opone a otra pareja que colabora entre sí en un espacio dividido por una red. La posibilidad de continuidad que permite el reglamento al poder jugar con los cristales, la reja metálica e incluso el juego exterior exige una alta capacidad de adaptación continua y toma de decisiones (Sánchez-Alcaraz, 2014). El rebote de un tipo u otro de pelota en función del efecto provocado por la pareja adversaria, que pueda venir de un cristal con más o menos humedad, 0 incluso del entramado metálico lleva, a menudo, a explorar diversas y creativas coordinaciones motoras funcionales. El éxito en el juego reside tanto más en la eficacia que en la eficiencia, es decir, en que el jugador o jugadora resuelva los problemas que van a aparecer continuamente, aunque no sea con el golpe más ortodoxo.

De ahí que nos planteemos la necesidad de desafiar a quien aprende, 0 a quien pretende que emerja un juego más efectivo, a explorar patrones coordinativos de forma dinámica y aceptando la no linealidad de los procesos, más allá de la mera repetición e imitación de la acción (Chow, Davids, Button \& Renshaw, 2015), y que tiendan a descubrir las respuestas más eficaces en función de sus características y del momento del entor- 
no y de la situación de juego en que se dan.

U na propuesta que ha surgido a partir de estas directices es la Constraints-Led Approach, y recientemente se han actualizado sus principios (Pol, Balagué, Ric, Torrents, Kiely \& H ristovski, 2020) de forma que puede ayudar aún más a adecuarse al entrenamiento del pádel. Por eso y todo lo explicado anteriormente, el objetivo de este artículo es presentar esta propuesta y describir aplicaciones específicas al entrenamiento del pádel, ya sea en la iniciación o en etapas posteriores. Más detalladamente, se presentarán dos experiencias didácticas aplicables a cualquier contexto de entrena miento de este deporte que se han estudiado de forma preliminar en diversos grupos de población.

\section{El ConstraintsLedApproachy su actualización teniendo en cuenta el anidamiento de constreñimientos}

El Constraints-Led Approach (en adelante CLA) es una propuesta de entrenamiento en sintonía con el marco conceptual de las ciencias de la complejidad, los sistemas dinámicos y la psicología ecológica (Davids et al., 2008). Las ciencias de la complejidad nos ofrecen principios de comportamiento que podemos observar y estudiar en el deporte y que transforman muchos de los postulados de las teorías de entrenamiento. Eso va más allá de una metodología concreta, puesto que muchas pueden ser válidas en función del contexto, y considera mos que lo más interesante de su puesta en práctica es el cambio de objetivos. El cambio de paradigma pone en duda conceptos como la adquisición de habilidades concretas o de condición física, para pasar a concepciones globales y a la búsqueda de personas con más capacidad de adaptación y con más posibilidades de presentar comportamientos diversos y funcionales, así como en focalizar el entrenamiento en los equipos como sistemas, y no en sumas de individualidades (Pol et al. , 2020). No obstante, las aplicaciones prácticas que se presentan en este artículo se centran en una metodología concreta, en la modificación de los constreñimientos de la ta rea, y se propone su uso justamente con el objetivo de conseguir, tanto en la iniciación como en el rendimiento deportivo, jugadoras y jugadores más imprevisibles para el rival, pero más establemente coordinados con sus parejas de juego, estableciendo como consecuencia sinergias funcional es (Passos, Lacasa, Milho \& Torrents, 2020). Ese camino no puede basarse exclusivamente en esta metodología, pero sí que podrá utilizarse en la pista como una buena herramienta.
La perspectiva en la cual se basa la CLA, que ofrece una explicación integrada para las respuestas adaptativas y variables del comportamiento deportivo, es principalmente la de las teorías de la Dinámica de la Coordinación de Scott Kelso (1995) y la Psicología Ecológica de James Gibson (1979). Entendemos, bajo esta perspectiva, queel comportamiento es un fenómeno de autoorganización que emerge de la continua interacción dinámica entre la persona que juega y las posibilidades, oportunidades 0 invitaciones a la acción (affordances) (Gibson, 1979) que el entorno ofrece a cada persona en cadasituación única. Un globo «llovido» posibilitaaunos a rematar para definir el punto, mientras que a otros tan solo les invita a mantener la bola al otro lado de la red. La confianza, el estado del marcador, la ubicación de la pareja contraria, la estabilidad previa al golpeo 0 el viento van a permitir (afford) acciones diferentes a personas diferentes en momentos diferentes. El juga dor actúa porque percibe, y al moverse genera un nuevo entorno que va a dar lugar a múltiples variaciones con nuevas posibilidades de acción inmediata. La percepción de la situación y la acción motriz están profundamente entrelazadas de tal forma que no se pueden entender si no es de forma contextualizada (Araújo, Teques, Hernández-M endo, Reigal \& Anguera, 2016).

Nos preguntamos cómo a pesar de ejecutar con eficacia una bandeja en una serie de repeticiones descontextualizadas en situación de entrenamiento, una deportista no consigue transferirla, a continuación, a la situación de juego contextual. Esto ocurre porque el comportamiento de la jugadora no se puede entender sin una referencia sostenida al contexto ambiental específico en el que surge (Renshaw, Davids, Shuttleworth $\&$ Chow, 2009). Esa emergencia es fruto de la autoorganización, y el aprendizaje o la mejora del rendimiento se produciráa medida que la jugadora interactúe con la información del entorno. El enfoque que adopta mos aquí se centra más en la naturaleza recíproca y simultánea de la percepción y la acción que en los supuestos- mecanismos de control, representaciones internalizadas y estructuras de conocimiento al macena das en la memoria (Woods, McKeown, Rothwell, Araújo, Robertson \& Davids, 2020).

Todas las dimensiones del entrenamiento van a estar interrelacionadas. Los procesos de adquisición de habilidades y de coordinación interpersonal (elementos protagonistas en el entrenamiento del pádel) se correlacionarán con el resto de elementos, como el entrenamiento de las capacidades físicas. Habrá una relación circular y anidada entretodos ellos, interactuando 
de abajo a arriba (un cambio celular modificará el comportamiento observable) y de arriba a abajo (un cambio en los valores enfatizados en el entrenamiento modificará los efectos del entrenamiento a nivel muscular), aunque actuarán a escal as temporales diferentes (Pol et al., 2020).

LaCLA se centra eminentemente en la persona que aprende, pero creemos que además es importante considerar que la persona que guía los procesos también forma parte del sistema de aprendizaje (Renshaw \& Brendan, 2018). 0 tro punto importante atener en cuenta es que en deportes colectivos como el pádel será importante centrarse en el sistema que forma la pareja, y que esa sea el objetivo de los procesos de cambio. Tanto la persona como la pareja llegarán al entrenamiento con una dinámica intrínseca que interactuará con los constreñimientos de la tarea. Serán esos los más modificables por parte del equipo técnico o del profesorado, aunque no losúnicos, y es en ellos en losque nos centraremos en las propuestas prácticas que presentamos.

\section{Aplicaciones al pádel}

Al aproximarnos al pádel bajo el paradigma de las ciencias de la complejidad asumimos que jugadores y equipos son sistemas adaptativos complejos con propiedades no lineales inherentes. A pesar de que a menudo se cree que va a existir una proporcionalidad entre la mejora del comportamiento técnico con el rendimiento deportivo, no es exactamente así. El aprendizaje está sujeto a diferentes fases que no siguen ni una linealidad ni unaproporcionalidad, y comprobamoscambioso transiciones sorprendentemente espectaculares combinados con periodos en qué quien aprende 0 entrena siente que no avanza. Una «extraordinaria técnica» puede ayudar en un momento dado a tener un resultado fantástico, y en el transcurso del mismo partido, una mala mirada de un compañero o el miedo a cerrar un partido pueden colapsar esa capacidad coordinativa y derivar en un estrepitoso fracaso. Hay en realidad una no-proporcionalidad entre habilidad técnica y rendimiento.

La jerarquía habitual también pierde fuerza cuando utilizamos el marco teórico de la complejidad. Se piensa, a menudo, que el cerebro se encarga de la completa organización, control y dirección del comportamiento de la misma forma que pensamos que el equipo técnico ejerce ese poder sobre el/ la jugador/ a. La realidad, en cambio, se encarga de ofrecernos muestras recurrentes de que quizá esa relación entre el cerebro y el compor- tamiento se modela de una formamenosjerárquica (Van O rden, Hollis \& Wallot, 2012). Es de la capacidad adaptativa y de auto-organización de un organismo que habita un entorno cambiante de la que va a emerger una acción funcional. Y con entorno cambiante ya no sólo nos referimos a aquellos aspectos del medio con los que iniciábamos estas reflexiones. Nuestro repertorio de gestos no está almacenado en el sistema nervioso central a la espera de ser escogido a voluntad. Es la relación de las posibilidades de acción y recursos de la persona en función de los constreñimientos impuestos por el entorno -que varían de forma continua y dinámica la que va a provocar la emergencia de comportamiento (Araújo \& Davids, 2011). De ahí que cuando optamos por un reduccionismo sistemático, eliminando elementos de la lógica interna del juego, debemos considerar el hecho de que estamos sustrayendo la posibilidad al aprendiz de la ontología mínima (individuo-ambiente) que conforma el rendimiento deportivo.

Todo aquel que practica un deporte de raqueta ha sentido al guna vez que ha fallado al guna bola «por pensar demasiado». Q ue quizás, durante la vorágine dinámica del juego, concentrado en estado de flow, se resuelven eficazmente jugadas... ¡sin saber exactamente cómo! No es imprescindible conocer o planificar las respuestas, estas emergerán espontáneamente sin necesidad de que alguien, desde dentro o desde fuera, lo ordene.

Abordar la iniciación o el rendimiento desde esta perspectiva sugiere que una función fundamental del profesorado o del equipo técnico es la de manipular constreñimientos clave para facilitar la emergencia y el descubrimiento de comportamientos funcionales (Renshaw et al., 2009). Se enfatiza la idea del jugador que trata de descubrir la solución al problema que se le propone, que conectaíntimamente con lanecesariatoma de decisiones y exige una actitud activa por parte del aprendiz (Martín-Barrero \& Camacho-Lazarraga, 2020). No solo debemos permitir, sino que debemos estimular al alumnado o deportistas a que exploren tantas formas como sean posibles para ser eficaces en la solución de un problema motor. Incluso considerar aquellas que no funcionen va a ser importantísimo en el proceso de aprendizaje, ya que los «errores» serán en realidad fluctuaciones del comportamiento necesarias para que se produzca el cambio (Caldeira, Paulo, Infante $\&$ Araújo, 2019). Debemos tratar, además, de que ese aprendizaje por descubrimiento ocurra en prácticas que estén en sintonía con el contexto de rendimiento para el que estamos preparando al deportista, puesto que le va a 
permitir ser más sensible a las fuentes de información disponibles y afinar, en base a la interacción, qué oportunidades de acción se van ofreciendo continuamente. La investigación nos muestra evidencias de que esa va riabilidad no constituye una desviación del gesto experto ideal que debemos corregir cuanto antes en los que se inician (Seifert, Orth, Button, Brymer $\&$ Davids, 2017). La variabilidad nos lleva a explorar cuál es el comportamiento adaptativo más apropiado en cada situación. Bajo este paradigma, la adaptabilidad se refiere a la adecuada relación entre comportamientos persistentes (estabilidad) y comportamientos variables (flexibilidad). Se pretende formar deportistas que exhiban patrones de comportamiento muy estables cuando sea necesario, pero capaces también de variarlos cuando la dinámica del juego lo exige. Aspecto de especial relevancia en el pádel en el que tanto penaliza el error no forzado. El entrenamiento de esa variabilidad de movimiento que va a soportar la adaptación, la sintetizaba magistralmente Nicolai Bernstein (1967) con el aforismo «repetition without repetition», nada menos que en la segunda mitad del siglo XX. El autor aludía así al hecho de que en lugar de «enseñar» el gesto técnico adecuado, lo que se debe practicar es la búsqueda de la solución motora óptima para cada situación.

Cualquier practicante de deportes de raqueta ha presenciado innumerables veces la escena del instructor con unos niños o adultos en fila esperando su momento para ejecutar la salida de pared de revés, 0 ejerciendo de modelo en la ejecución de golpeos de pádel como la bandeja sin pelota por medio. En esos ejemplos, con el afán de facilitar la tarea, se cae en la descomposición y la descontextualización. Comprender los elementos informativos clave específicos de cada deporte que contribuyen a regular el comportamiento nos va a permitir diseñar tareas de aprendizaje representativas que mantengan 0 se aproximen a las demandas reales (Cantos \& M oreno, 2019). Por ejemplo, mantener la estructura de cancha dividida y de colaboración y oposición en espacio y tiempo alternativo nos parecen imprescindibles para generar situaciones de enseñanza aprendizaje 0 de entrenamiento que permitan la emergencia de comportamientos que sirvan para que los jugadores o las jugadoras sean más funcional mente diversos. Si el juego del pádel es una actividad de una exigencia informativa altísima, ino deberíamos también plantear escenarios ricos en posibilidades de acción en nuestras clases y entrenamientos? Diversos estudios sobre la práctica con tareas no representativas ponen en duda la transferibilidad de los patrones de movimientos de- sarrollados en los ejercicios descontextualizados a los entornos de rendimiento (Pinder, Davids \& Renshaw, 2012).

En los deportes de raqueta existen al gunos estudios basados en esta perspectiva, en los cuales se manipuló el equipamiento deportivo. En el tenis, por ejemplo, se ha manipulado la altura de la red y las dimensiones de la pista para mejorar las oportunidades de aprendizaje (Limpens, Buszard, Shoemaker, Savelsbergh \& Reid, 2018; Bayer, Ebert \& Leser, 2017). O tras modificaciones que se utilizan, aunque no existen estudios al respecto, son el uso de raquetas de diferentes materiales y dimensiones, incluir pelotas de presión variada, indiacas o globos, proponer redes de doble al tura, de paso varia ble 0 el uso de «zona muerta». Existe, hoy en día, una gran variedad de opciones comerciales de palas y pelotas adaptadas. Aún así, no es difícil ver niñas y niños de temprana edad en las escuelas de pádel con palas pesa das que no facilitan su manejo, utilizando una pelota reglamentaria y jugando en campo y reglamento adulto. Es ampliamente reconocido en la literatura del desa rrollo motor que debemos superar la idea de que el niño es un adulto en pequeño (Davids, Araújo, Correia \&Vilar, 2013).

Es competencia del profesorado o de los equipostécnicos adecuar los diseños de las tareas a las características y necesidades de un niño o niña de categoría benja mín, por ejemplo. Si bien existen al gunas iniciativas muy loables en el diseño de pistas adaptadas, es lógico pensar que las cuestiones financieras y de espacio suponen un problema para los clubes difícil de superar. Por ese motivo, a continuación, se presentan dos experiencias que permiten modificar la tarea, adaptándola a las necesidades del proceso de enseñanza-aprendizaje y entrenamiento, sin que suponga un sobrecoste para las entidades deportivas.

\section{Las situaciones reducidas (small-sided games) como constreñimiento de la tarea en el aprendizaje y entrenamiento del pádel}

Se presentan a continuación dos estudios piloto al respecto del impacto de la manipulación de constreñimientos de la tarea sobre el juego de pádel. El primero (el pekkepadel) pone el foco en el desarrollo de practicantes funcional mente diversos en la iniciación del pádel. El segundo (el cruzadito) pone el énfasis en comprender los cambios fisiológicos y cinemáticos que pueden provocar las situaciones reducidas en pádel. 


\section{El pakepadd: pádd ackptadba niñes yniños en su procesode aprendizaje}

El objetivo del estudio consistía en analizar el efecto de escalar el espacio de juego, móvil e implemento en el comportamiento de niñas y niños.

\section{Metodología}

El estudio comparó el comportamiento mostrado por 8 jugadoras y jugadores de la escuela de menores (5 niños y 3 niñas, edad: $7.6 \pm 0.7$ ) disputando sendos partidos a nueve juegos. El primero con pala, pelota y dimensión del campo habitual (20x10 m), mientras que el otro en un campo de pádel adaptado (10×6 m) con una red portátil perpendicular al cristal de fondo, pala de $33 \mathrm{~cm}$ y pelota softl. Se registró en vídeo los partidos con una cámara GoPro Hero 3 full HD y gran angular. Se utilizó un instrumento de observación validado (Almonacid-Cruz, 2011) que agrupa los diferentes golpeos habituales del juego en cuatro categorías: golpes de fondo, de pared, de red y de definición. Para el análisis observacional se utilizó un software específico de análisis observacional (Longomatch Pro v.1.2.2., Fluendo, Barcelona, España). Después de un proceso de entrenamiento del observador (Licenciado Ciencias de laActividad Física y el Deporte y monitor en activo de la escuela de pádel) se garantizó una fiabilidad del $95 \%$ de coincidencia de las observaciones realizadas con una semana de diferencia. Tras proceder a la codificación de los cuatro partidos, se exportaron los datos con el fin de tratamiento estadístico. Se empleó la prueba SaphiroWilk para comprobar la distribución normal de cada una de las variables. A partir de los resultados obtenidos en la prueba de normalidad, con las variables fondo, pared y red, se utilizó la prueba paramétrica t de Student de muestras relacionadas; y para la variable definición se realizó la prueba no paramétrica de Wilcoxon. Se esta bleció un nivel de significación de $p<$. 05. Todos los análisis de datos se real izaron con el paquete estadístico SPSS v. 23.0 paraW indows (SPSS Inc., Chicago, IL, EUA).

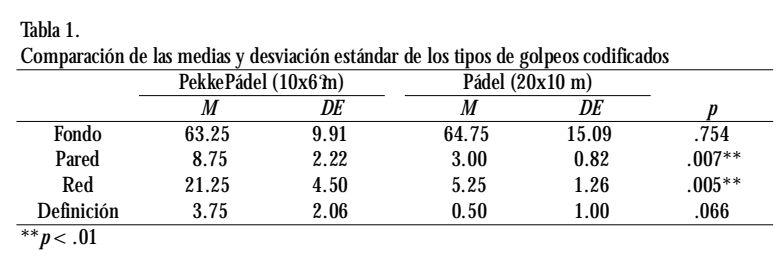

Resultados y discusión

En la tabla 1 se muestra un resumen de los golpeos agrupados en las categorías citadas anteriormente.
El análisis de los datos muestra la emergencia de un mayor número de golpeos en tres de las cuatro categorías analizadas al competir en el formato del juego reducido. Un espacio reducido ofrece la oportunidad de aproximarse a la red (adaptada también al tamaño de los jóvenes jugadores) y tratar de llevar la iniciativa del juego. Una pala más ligera y más pequeña ofrece una nueva gama de posibilidades de coordinación de movimientos, hasta el punto de que se atrevieran incluso con acciones de contrapared y contrapared lateral que difícilmente el espacio reglamentario les hubiera permitido practicar. La manipulación de las dimensiones del campo, aś como la reducción de tamaño y peso de pala y pelota, posibilitan oportunidades de acción diversas y creativas.

\section{El arzadita unjuagoreduacbaruzadb}

El objetivo del estudio pretendía comparar los requerimientos fisiológicos, cinemáticos y neuromusculares específicos del pádel en participantes de diferentes edades entre un juego reducido común en el pádel, el juego 1vs. 1 cruzado, y el juego reglamenta rio.

\section{Metodología}

Participaron en el estudio 12 jugadores de pádel amateur divididos en tres grupos de edad: 4 menores (14.25 \pm 1.50$), 4$ adultos ( $39.50 \pm 8.50)$ y 4 veteranos $(63.33 \pm 4.51)$. Jugar on 12 minutos en dos condiciones diferentes: a) situación reglamentaria y b) juego modificado. El juego modificado consistía en jugar solo en medio campo cruzado y con las reglas habituales de pádel. Los jugadores Ilevaron dispositivos inerciales W IMU ${ }^{\top M}$ adaptados mediante un chaleco y un receptor de frecuencia cardíaca ajustado al pecho (RealTrack System, Almería, España) con el fin de registrar variablescinemáticas, fisiológicasy neuromusculares. Molina Carmona, Gómez-Carmona, BastidaCastillo y PinoO rtega (2018) han constatado la validez y fiabilidad del dispositivo para este tipo de registros. Concretamente las variables susceptibles de análisis fueron la frecuencia cardíaca, la distancia total recorrida, aceleraciones, desaceleraciones, velocidades máximas, mínima y carga de trabajo (playerload).

Las variables se resumieron mediante la mediana (Mdn) y el intervalo intercuartil (IQI), y se compararon antes y después de jugar mediante dos pruebas no paramétricas: la estimación del intervalo de confianza de la mediana de la diferencia ( $\mathrm{M} \mathrm{d} 95 \% \mathrm{Cl}$ ) y la prueba de aleatorización de Fisher-Pitman (p). 
Tabla 2.

Comparación de las diferentes variables cinemáticas, fisiológicas y neuromusculares de los participantes en situación de partido habitual respecto del juego reducido y condicionado.

\begin{tabular}{|c|c|c|c|c|c|c|c|}
\hline \multirow[b]{2}{*}{ Variable } & \multicolumn{2}{|c|}{ Pista total $(20 \times 10 \mathrm{~m})$} & \multicolumn{2}{|c|}{ M edia pista cr uzada } & \multirow[b]{2}{*}{$M d$} & \multirow[b]{2}{*}{$95 \% \mathrm{Cl}$} & \multirow[b]{2}{*}{$p$} \\
\hline & Mdn & $|Q|$ & $M d n$ & $\mid \mathrm{IQI}$ & & & \\
\hline FC media (Ipm) & 121,0 & {$[113,0 ; 161,0]$} & 131,5 & {$[122,0 ; 172,0]$} & 11,0 & {$[0,5 ; 19,5]$} & 0,005 \\
\hline FC máx (Ipm) & 144,0 & {$[130,0 ; 184,0]$} & 154,0 & {$[140,0 ; 189,0]$} & 6,5 & {$[-5,5 ; 15,0]$} & 0,035 \\
\hline PL min (a.u) & 0,53 & {$[0,49 ; 0,68]$} & 0,67 & {$[0,61 ; 0,75]$} & 0,13 & {$[0,03 ; 0,23]$} & 0,002 \\
\hline Dist cubierta (m) & 511,8 & {$[454,1 ; 587,0]$} & 643,0 & {$[577,8 ; 666,6]$} & 111,8 & {$[70,4 ; 166,4]$} & 0,001 \\
\hline DistCub/ $\mathrm{min}(\mathrm{m} / \mathrm{min})$ & 41,5 & {$[39,8 ; 48,1]$} & 50,8 & {$[48,0 ; 55,2]$} & 9,4 & {$[6,3 ; 13,1]$} & 0,001 \\
\hline Número Acel & 285,0 & {$[261,0 ; 333,5]$} & 336,0 & {$[315,0 ; 361,0]$} & 59,0 & $9,0]$ & 0,006 \\
\hline Número Decelerac & 284,5 & {$[260,5 ; 332,5]$} & 336,0 & {$[314,0 ; 361,0]$} & 58,5 & {$[-2,5 ; 79,0]$} & 0,006 \\
\hline Núm. Acel/ min & 23,5 & {$[21,7 ; 25,6]$} & 27,8 & {$[26,0 ; 28,8]$} & 5,0 & {$[-0,1$} & 0,005 \\
\hline Núm. Decel/ min & 23,5 & {$[21,6 ; 25,6]$} & 27,8 & {$[26,0 ; 28,8]$} & 4,9 & {$[-0,1$} & 0,004 \\
\hline Acel máx & 3,5 & {$[3,2 ; 3,8]$} & 3,2 & {$[2,9 ; 4,0]$} & $-0,03$ & {$[-0,74 ; 0,76]$} & 0,644 \\
\hline DCC max & $-3,0$ & {$[-3,5 ;-2,7]$} & $-3,0$ & {$[-3,5 ;-2,9]$} & $-0,04$ & {$[-0,65 ; 0,46]$} & 0,366 \\
\hline Vel med $(\mathrm{km} / \mathrm{h})$ & 2,9 & {$[2,9 ; 3,1]$} & 3,3 & {$[3,1 ; 3,5]$} & 0,32 & {$[0,21 ; 0,54]$} & 0,001 \\
\hline Vel máx $(\mathrm{km} / \mathrm{h})$ & 12,0 & {$[11,4 ; 12,7]$} & 12,0 & {$[11,43 ; 13,16]$} & 0,28 & {$[-1,1 ; 0,97]$} & 0,679 \\
\hline Cantidad Impactos & 39,5 & {$[32,0 ; 64,0]$} & 56,0 & {$[37,0 ; 99,0]$} & 5,0 & {$[-4,5 ; 68,5]$} & 0,049 \\
\hline Player load (a.u) & 6,6 & {$[5,9 ; 8,2]$} & 8,7 & {$[7,2 ; 9,2]$} & 1,5 & {$[0,35 ; 3,4]$} & 0,002 \\
\hline
\end{tabular}

\section{Resultados y discusión}

Lamanipulación de constreñimientos delatarealleva consigo adaptaciones del comportamiento del jugador. Jugar con la mitad de espacio podía parecer, a priori, que la demanda de esfuerzo físico, respecto la exigencia del juego reglamentario, se debía reducir también. Los resultados del estudio que se muestran en la tabla 2 indican que el impacto sobre la demanda fisiológica del jugador no sólo no fue menor, sino que, en la mayoría de las variables anal izadas, lo incrementó ligeramente. Esta tendencia se muestra en consonancia con otros estudios (C asamichana\& Castellano, 2010; D ellal, 0 wen, Wong, Krustrup, Van Exsel \& Mallo, 2012) en que también comparaban variables fisiológicas y cinemáticas entre situaciones de juego reducido y juego reglamentario en fútbol. Los autores constataron además en sus conclusiones la necesidad de contar con las evidencias de este tipo de estudios para que los equipos técnicos comprendan las diferentes demandasfisiológicasimpuestas a los jugadores al manipular los constreñimientos de la tarea. Así lo aseveran también Sánchez-Alcaraz, O rozco-Ballesta, Courel-Ibáñez y Sánchez-Pay (2018) en uno de los primeros estudios que ha eval uado la condición física en jugadores jóvenes en pádel a través de diversos test de condición física.

En nuestro trabajo, las variables de frecuencia cardíaca media y máxima reportaron valores de exigencia mayores en el juego reducido que en la situación competitiva en espacio reglamentario en los tres grupos de población. Lo mismo sucedió con distancias recorridas, aceleraciones, deceleraciones, velocidad, impactos y carga total de trabajo. Este hecho sugiere que la modificación de los constreñimientos de la tarea en el sentido que plantea este estudio se trata de una interesante herramienta para entrenar respetando la interacción anidadade todos losfactoresque intervienen en el aprendizaje y rendimiento deportivo.
Como no puede ser de otra forma, el estudio nos genera nuevas cuestiones y curiosidades acerca de cómo van a cambiar estos valores a medida que podamos incorporar nuevos constreñimientos en formas de nuevas reglas u otros tipos de limitaciones. La literatura sugiere que al alterar esos factores va a variar la carga de trabajo percibida por los jugadores, así como sus respuestas fisiológicas, tácticas y técnicas (Halouani, Chtourou, Gabbett, Chaouachi \& Chamari, 2014). Es evidente que necesitamos estudiarlas mucho más para comprender esas interacciones y escoger aquellos constreñimientos de la tarea susceptibles de mejorar los procesos de aprendizaje y rendimiento de nuestras/ os jugadoras/ es.

\section{Conclusiones}

Consideramos que el entrenamiento requiere una reflexión profunda sobre sus objetivos y sobre la comprensión de los fenómenos que emergen durante cualquier proceso de cambio, con el fin de poder diseñar tareas más adecuadas a dichos objetivos. El constraintsled approach es una propuesta que está en sintonía con el paradigma de la complejidad, asumiendo los procesos de auto-organización, la sensibilidad en la elección de constreñimientos y formas de manipulación, el rigor en el diseño de aprendizajes representativos o la creatividad parainfundir la variabilidad necesaria. Esta propuesta se basa en la psicología ecológica y en el acoplamiento percepción-acción, y requiere escucha activa con todas las personas que intervienen en los procesos de cambio, el conocimiento de los principios operativos del juego y la comprensión de las capacidades individuales de alumnado o deportistas. Además, la propuesta se ha actualizado teniendo en cuenta la causal idad anidada y circular entre todos los constreñimientos.

La reducción de las dimensiones del campo y del equipamiento en la iniciación, o la modificación de la estructura espacial en cualquier etapa deportiva parecen dos propuestas válidas para conseguir una mayor diversidad funcional en jugadoras y jugadores de pádel, pudiendo conseguir, incluso, una mayor exigencia física del juego.

\section{Agradecimientos}

Este estudio ha sido posible gracias a los recursos que el Instituto Nacional de Educación Física de Cataluña (INEFC) destina a la investigación, así como a Ekke- 
Viding al facilitarnos acceso al conjunto de la sección de pàdel.

\section{Referencias}

Aivar, M.P., \& Ramon, T. (2006). La dualidad percepción-acción: la necesidad de recuperar la crítica de Dewey a la teoría del arco reflejo. Revista de Historia de la Psicología, 27 (2/ 3), 269-277.

Almonacid-Cruz, B. (2011). Perfil de juego en pádel de al to nivel. (Tesis doctora). Jaén: Universidad de Jaén.

Araújo, D., \& Davids, K. (2011). W hat Exactly is Acquired During Skill Acquisition? Journal of Consciounness Studies, 18(4), 7-23.

Araújo, D., Teques, P., Hernández-Mendo, A., Reigal, R., \& Anguera, M.T. (2016). La toma de decisión, ies una conducta observable?: Discusión sobre diferentes perspectivas teóricas utilizadas en el estudio del rendimiento deportivo. Cuadernos de psicología del deporte, 16(1), 183-196.

Balagué, N., Pol, R., Torrents, C., Ric, A., \& Hristovski, R. (2019). On the relatedness and nestedness of contraints. Sports M edicine 0 pen, 5(1), 110. https:/ / doi.org/ 10.1186/ s40798-019-0178-z

Bayer, D., Ebert, M., \& Leser, R. (2017). A comparison of the playing structure in elite kids tennis on two different scaled courts. International Journal of PerformanceAnalysisin Sport, 17 (1-2), 34-43. http:/ / dx. doi.org/ 10.1080/24748668.2017.1303977

Bernstein, N.A. (1967) The coordination and regulation of movements Oxford: Pergamon Press.

Caldeira, P., Paulo, A., Infante, J., \& Araújo, D. (2019). A influência da pedagogia não-linear e da abordagem baseada nos constrangimentos no treino do remate no voleibol. Retos: Nuevas Tendencias en Educación Física, Deporte y Recreación, (36), 590-596

Cantos, J., \& Moreno, F. J. (2019). Pedagogía no lineal como método de enseñanza de los comportamientos tácticos en los deportes de equipo, aplicación al rugby. Retos: NuevasTendencias en Educación Física, Deporte y Recreación, (35), 402-406.

Casamichana, D. \& C Castellano, J. (2010). Time- motion, heart rate, perceptual and motor behaviour demands in small-sides soccer games: Effects of pitch size. Journal of Sports Sciences, 28(14), 1615-1623. http:/ / dx. doi.org/ 10.1080/ 02640414.2010.521168

Chow, J.Y., Davids, K., H ristovski, R., A raújo, D., \& Passos, P. (2011). N onlinear pedagogy: Learning design for self-organizing neurobiological systems. N ew Ideas in Psychology, 29(2), 189-200. http:/ / dx. doi.org/ 10.1016/ j. newideapsych. 2010.10.001

Chow, J.Y., Davids, K., Button, C., \& Renshaw, I. (2015). N onlinear Pedagogy in Skill Acquisition: An introduction. New York:Taylor\&Francis Group. http:/ / dx. doi. org/ 10.4324/ 9781315813042

Davids, K., Araújo, D., Correia, V., \& Vilar, L. (2013). How small-sided and conditioned games enhance acquisition of movement and decisionmaking skills. Exercise and Sport Sciences Reviews, 41(3), 154-161. http:/ / dx. doi.org/ 10.1097/ JES.0b013e318292f3ec

Davids, K., Button, C., \& Bennett, S. (2008). Dynamics of skill acquisition: A consraints-led approach. Human Kinetics.

Dellal, A., O wen, A., Wong, D. P., Krustrup, P., Van Exsel, M., \& Mallo, J. (2012). Technical and physical demands of small vs. large sided games in relation to playing position in elite soccer. Human movement science, 31(4), 957-969. http:/ / dx. doi.org/ 10.1016/ j. humov.2011.08.013

Gibson, J. J. (1979). The Ecological Approach to Visual Perception. Boston: Houghton Mifflin

Halouani, J., Chtourou, H., Gabbett, T., Chaouachi, A., \& Chamari, K. (2014). Small-sided games in team sports training: a brief review. The Journal of Strength \& Conditioning Research, 28(12), 3594-3618. http:/ / dx. doi.org/ 10.1519/ JSC.0000000000000564

Headrick, J., Renshaw, I., Davids, K., Pinder, R. A., \& Araújo, D. (2015). The dynamics of expertise acquisition in sport: The role of affective learning design. Psychology of Sport and Exercise, 16, 83-90. http:// dx. doi.org/ 10.1016/ j. psychsport.2014.08.006

Heras Escribano, M. (2012). Comprender la realidad sin representaciones: Affordances y psicología ecológica. Ciencia Cognitiva, 6(2), 48-50.
Kelso, J. A. S. (1995). Dynamic patterns: the seff-organisation of brain and behaviour. Cambridge, MA: MIT Press.

Limpens, V., Buszard, T., Shoemaker, E., Savelsbergh, G. J., \& Reid, M. (2018). Scaling constraints in junior tennis: the influence of net height on skilled players' match-play performance. Research quarterly for exercise and sport, 89(1), 1-10. http:// dx.doi.org/ 10.1080/ 02701367.2017.1413230

Martín-Barrero, A., \& Camacho-Lazarraga, P. (2020). El diseño de tareas de entrenamiento en el fútbol desde el enfoque de la pedagogía no lineal. Retos: NuevasTendencias en Educación Físca, Deporte y Recreación, (38), 768772.

Mitchell, S.A., O slin, J.L., \& Griffin, L.L. (2006). Teaching sports skills: A tactical games approach ( $2^{\text {nd }}$ ed.). Champaign, IL: Human Kinetics.

Molina-Carmona, I., Gómez-Carmona, C. D., Bastida Castillo, A., \& PinoOrtega, J. (2018). Validez del dispositivo inercial W IMU PRO ${ }^{\mathrm{TM}}$ para el registro de la frecuencia cardíaca en un test de campo. Sportk Revista Euroamericana de Ciencias del Deporte, 7, 81-86.

Newell, K. M. (1986). Constraints on the development of coordination. En M. G. Wade\& H. T. A.W hiting (Eds), Motor skill acqui istion in children. Aspects of coordination and control, (pp. 341-360). Amsterdam: Martinus Nijhoff.

Passos, P., Araújo, D., Davids, K., \& Shuttleworth, R. (2008). Manipulating constraints to train decision making in rugby union. International Journal of Sports Science and Coaching, 3(1), 125-140. http:/ / dx. doi.org/ $10.1260 / 174795408784089432$

Passos, P., Lacasa, E., Milho, J., \& Torrents, C. (2020). Capturing Interpersonal Synergies in Social Settings: An Example within Badminton Cooperative Task. Nonlinear Dynamics, Psychology, and Life Sciences, 24 (1), 59-78.

Pinder, R. A., Davids, K., \& Renshaw, I. (2012). Metastability and emergent performance of dynamic interceptive actions. Journal of Science and Medicine in Sport, 15(5), 437-443. http:/ / dx. doi.org/ 10.1016/ j.jsams. 2012.01.002

Pol, R., Balagué, N., Ric, A., Torrents, C., Kiely, J., \& Hristovski, R. (2020). Training or Synergizing? Complex Systems Principles Change the Understanding of Sport Processes. Sports Medicine-Open, 6(1), 1-13. https: / / doi.org/ 10.1186/ s40798-020-00256-9

Renshaw, I., Davids, K. W., Shuttleworth, R., \& Chow, J.Y. (2009). Insights from ecological psychology and dynamical systems theory can underpin a philosophy of coaching International Journal of Sport Psychology, 40(4), 540-602.

Renshaw, I., \& Brendan, M. (2018). A Constraint-LedApproach to Coaching and Teaching Games: $C$ an going back to the future solve the «they need the basics before they can play a game» argument? Ágora para la Educació Física y el Deporte, 20(1), 1-26. http:// dx.doi.org/ 10.24197/ aefd.1.2018.1-26

Sánchez-Alcaraz, B.J. (2014). Diferencias en las acciones de juego y la estructura temporal entre el pádel masculino y femenino profesional. Acción Motriz, 12, 17-22

Sánchez-Alcaraz, B., O rozco-Ballesta, V., Courel-Ibáñez, J., \& Sánchez-Pay, A. (2018). Evaluación de la velocidad, agilidad y fuerza en jóvenes jugadores de pádel. Retos: Nuevas Tendencias en Educación Físca, Deporte y Recreación, (34), 263-266.

Seifert, L., O rth, D., Button, C., Brymer, E., \& Davids, K. (2017). An ecological dynamics framework for the acquisition of perceptual-motor skills in climbing. En F. Feletti (Ed.), Extreme Sports M edicine, (pp. 365-382). Springer, Cham. http:/ / dx.doi.org/ 10.1007/ 978-3-31928265-7_28

Van O rden, G., Hollis, G., \&Wallot, S. (2012). The blue-collar brain. Frontiers in physiology, 3, 207. http:/ / dx.doi.org/ 10.3389/ fphys. 2012.00207

Woods, C., Mckeown, I., Rothwell, M., Araújo, D., Robertson, S., \& Davids, K. (2020). Sport practitioners as sport ecology designers: How ecological dynamics has progressively changed perceptions of skill'acquisition'in the sporting habitat. Frontiers in Psychology, 11, 654. http:/ / dx. doi.org/ 10.3389/ fpsyg. 2020.00654 